\title{
Minimal Clinically Important Differences (MCID) for the Western Ontario Osteoarthritis of the Shoulder Index (WOOS) and the Oxford Shoulder Score (OSS)
}

\section{Marc Randall Kristensen \\ Nyring (D) \\ Bo Sanderhoff Olsen (iD \\ Alexander Amundsen (D) \\ Jeppe Vejlgaard Rasmussen (iD}

Department of Orthopedic Surgery, Herlev and Gentofte Hospital, Hellerup, 2900, Denmark
Correspondence: Marc Randall

Kristensen Nyring

Department of Orthopedic Surgery,

Herlev and Gentofte Hospital, University

of Copenhagen, Gentofte Hospitalsvej 12,

Hellerup, 2900, Denmark

Tel +45 38673840

Email marc.randall.kristensen.

nyring@regionh.dk
Background: The minimal clinically important difference (MCID) is an important instrument in the interpretation of changes in patient-reported outcome measures (PROM). To our knowledge, no MCID of the Western Ontario Osteoarthritis of the Shoulder Index (WOOS) score has ever been reported and no studies have reported an MCID for the Oxford Shoulder Score (OSS) based on patients with glenohumeral osteoarthritis, treated with an anatomical total shoulder arthroplasty (aTSA). The aim of this study was to determine MCID for WOOS and OSS in a cohort of patients with glenohumeral osteoarthritis treated with an aTSA.

Methods: All patients treated with an aTSA for glenohumeral osteoarthritis at our institution between March 2017 and February 2019 were included. Each patient completed the WOOS and the OSS preoperatively and one year postoperatively. At one year, the patients were asked to rate their overall improvement on a 7-point scale. We used an anchor-based method as our primary method to calculate the MCID, supported by two different distribution-based methods.

Results: A total of 45 primary aTSA were included. The MCID of WOOS was 12.3 according to the anchor-based method and 14.2 and 10.3 according to the two distribution-based methods. The MCID of OSS was 4.3 according to the anchor-based method and 5.8 and 4.3 according to the two distribution-based methods.

Conclusion: The anchor-based method is considered superior to the distribution-based method, and therefore we advocate to use this as MCID. For patients with glenohumeral osteoarthritis treated with an aTSA, the MCID values were 12.3 points for WOOS and 4.3 points for OSS. To our knowledge, this is the first study to report a MCID value for WOOS and the first study to report a MCID value for OSS in this subgroup of patients.

Keywords: minimal clinically important difference, glenohumeral osteoarthritis, anatomical total shoulder arthroplasty, patient reported outcome measures

\section{Introduction}

Patient reported outcome measures (PROM) are a popular instrument in measuring the effect of a given treatment. PROMs are defined as self-evaluated measurements of any aspect of a patient's health status, without interpretation of the patient's response by a clinician or anyone else. ${ }^{1}$ PROMs can be used to assess a treatment effect in both research and clinical settings by comparing the baseline and the follow-up scores. However, the interpretation of change in scores is difficult if the 
clinical relevance is unknown. The minimal clinically important difference (MCID) can be used in the interpretation of changes in PROM scores. The MCID is defined as the smallest meaningful change in a health domain that a patient would identify as important. ${ }^{2}$

The use of anatomical total shoulder arthroplasty (aTSA) for glenohumeral osteoarthritis has increased in recent years, ${ }^{3}$ and PROMs are often used to monitor and evaluate the effect. ${ }^{4}$ Two frequently used PROMs are the Western Ontario Osteoarthritis of the Shoulder Index (WOOS $^{3,5}$ and the Oxford Shoulder Score (OSS). ${ }^{6,7}$

The WOOS and OSS are also used to monitor the results of shoulder arthroplasty on a national level by the Danish Shoulder Arthroplasty Register, ${ }^{8}$ the Swedish Shoulder Arthroplasty Register, ${ }^{9}$ the Australian Orthopaedic Association National Joint Replacement Registry, ${ }^{10}$ the British National Joint Registry, ${ }^{11}$ the New Zealand Joint Registry $^{12}$ and the Dutch Arthroplasty Register. ${ }^{13}$

To our knowledge, the MCID of WOOS has never been reported, which is confirmed in a recent systematic review. ${ }^{14}$ However, Razmjou et al reported the substantial clinical benefit (SCB) of WOOS, which, in contrast to MCID, is defined as the improvement in score that provides a substantial clinical benefit to the patient. ${ }^{15}$

The MCID of OSS has only been reported in four studies. ${ }^{2,16-18}$ One study ${ }^{16}$ reported the MCID for OSS based on patients with inflammatory or degenerative rheumatic disease treated with either TSA, hemiarthroplasty, synovectomy or subacromial bursectomy. One study ${ }^{17}$ included patients with a wide range of diagnoses and both operative and nonoperative treatment. Two studies ${ }^{2,18}$ focused on nonoperative treatment in patients with subacromial impingement syndrome and rotator cuff disease, respectively.

Obviously, due to the above, there is a large gap in the orthopaedic literature regarding the MCID for WOOS in general and the MCID for OSS based on patients with glenohumeral osteoarthritis treated with an aTSA. Therefore, it would be of great future benefit to all clinicians and researchers using these PROMs if the MCID for WOOS and OSS was determined in a cohort of patients with glenohumeral osteoarthritis treated with an aTSA, which was the aim of this study.

\section{Materials and Methods}

\section{Outcome Measures}

The Western Ontario Osteoarthritis of the Shoulder index (WOOS) is a disease-specific patient-reported outcome. ${ }^{19}$
There are 19 questions divided into four domains: Physical symptoms, sports and work, lifestyle and emotions. Each question is answered on a visual analogue scale ranging from 0 to 100 . The overall score ranges from 0 to 1900 , with 1900 being the worst. For ease of interpretation, the scores are often converted to a percentage of the maximum score. For the present we used the Danish version of WOOS which was translated according to international guidelines ${ }^{20}$ and validated using classical test theory in a cohort of patients treated with shoulder arthroplasty for osteoarthritis. $^{21}$

The Oxford Shoulder Score (OSS) was conceived as a measurement tool for the assessment of pain and function after elective shoulder surgery. ${ }^{22}$ There are 12 questions with each item scored from 0 to 4 . The overall score ranges from 0 to 48 , with 48 being the best. We use a Danish version of OSS which was translated according to international guidelines and validated using classical test theory. ${ }^{23}$ The validation was performed primarily on patients with painful rotator cuff disease.

\section{Patients}

We used a consecutive series of 58 Global Unite Anatomical Shoulder Arthroplasties (DePuy Synthes, Raynham, Massachusetts, United States of America) which was used for primary osteoarthritis. Each arthroplasty was included as independent cases. All procedures were performed by one of seven experienced shoulder surgeons between March 2017 and February 2019 at Herlev/Gentofte University Hospital, Denmark. The following inclusion and exclusion criteria were met in the enrolment of patients:

\section{Inclusion Criteria}

1. Primary glenohumeral osteoarthritis independent of previous joint preserving surgery,

2. Osteoarthritis on plain radiographs with standard anterior-posterior and lateral projections,

3. Insufficient effect of non-surgical treatment with symptoms severe enough to justify shoulder arthroplasty,

4. ASA scores 1-3, physically fit for surgery and rehabilitation.

\section{Exclusion Criteria}

1. Below 18 years of age,

2. Cognitive or linguistic impairment, 
3. Rotator cuff insufficiency defined as rotator cuff lesions or grade 2 fat infiltrations on MRI according to the Goutallier classification ${ }^{24,25}$ verified with impaired functional strength and perioperative findings,

4. Insufficient glenoid bone-stock or large $(>1 \mathrm{~cm})$ humeral bone cysts on CT verified with perioperative findings,

5. ASA scores $4-5$.

Each patient was asked to complete the WOOS and the OSS preoperatively and one year postoperatively. In one year, the patients were asked to rate their overall improvement on a 7-point scale. The following question was asked: What is the function of your shoulder today, compared to before your surgery? With the following answer options: (1) disaster, (2) much worse, (3) slightly worse, (4) unchanged, (5) slightly better (6) much better, (7) recovered. Afterwards, the answers were divided into the following groups: (1) patients who indicated that they were slightly worse, unchanged or slightly better were classified as not importantly changed, and (2) patients who indicated that they were much better or recovered were classified as importantly improved. The answer options disaster and much worse are categorized as importantly worsened and excluded from the anchorbased analysis, however, no patients reported this (Table 1).

\section{Statistics}

In general, two different approaches are used to determine the MCID. The anchor-based method uses an external variable and then examine the relation between this variable and the PROM. The distributionbased method is based on the statistical characteristics of the obtained PROM scores. ${ }^{26}$ It has been recommended by Revicki et $\mathrm{al}^{27}$ to use an anchor-based

Table I Distribution of Self-Rated Improvement

\begin{tabular}{|l|c|c|}
\hline & Patients & $\%$ \\
\hline Disaster & 0 & 0 \\
Much worse & 0 & 0 \\
Slightly worse & 2 & 5 \\
Unchanged & 1 & 2 \\
Slightly better & 10 & 24 \\
Much better & 22 & 52 \\
Recovered & 7 & 17 \\
\hline
\end{tabular}

method as primary evidence for MCID and a distribution-based method to provide secondary evidence for that MCID.

The primary method in this study was the anchor-based method which includes patient's own assessment of improvement, resulting in an extra dimension in the calculations. The anchor-based method we used, combines patients' own experience of improvement, in terms of their response to the anchor question, with their reported change in PROM scores. This is a frequently used method $^{28-32}$ where the MCID is calculated as the difference in mean improvement between the not importantly changed group and the importantly changed group defined by the anchor question.

To support the results of the anchor-based method, we used two different distribution-based methods. The first method is based on the standard error of measurement (SEM): ${ }^{33}$

$$
S E M=S D \cdot \sqrt{1-r}
$$

where one SEM is equivalent to the MCID. ${ }^{34} \mathrm{SD}$ is the standard deviation of the baseline scores and $r$ is a measure of reliability, represented by the Cronbach's $\alpha$.

The second distribution-based method is based on the mean standard deviation of the SD values of the PROM scores at baseline, follow-up and the difference between baseline follow-up. ${ }^{35}$ The half of this mean standard deviation has been proven to be a reasonable estimate of MCID in a systematic review by Norman et al. $^{36}$

$$
M C I D=0.5 \cdot \frac{\mathrm{SD}(\text { baseline })+\mathrm{SD}(\text { followup })+\mathrm{SD}(\text { difference })}{3}
$$

We reported mean values. This is considered reasonable, as the preoperative scores are approximately normally distributed, while the postoperative scores are rightskewed (a tendency for more values at the high end of the score), but with no major outliers.

SPSS was used for the statistical analysis (IBM Corp, Armonk, NY, USA). The level of statistical significance was set at $p<0.05$ and $p$-values were 2-tailed.

\section{Results}

Data of 58 primary aTSA were reviewed. 8 patients declined to participate in the 1-year follow-up and 5 patients were lost to follow-up. 3 patients had an incomplete WOOS and 2 patients had an incomplete OSS. This leaves 42 patients for the distribution-based analyses based on WOOS and 43 patients for the distribution-based analyses based on OSS (Figure 1). 


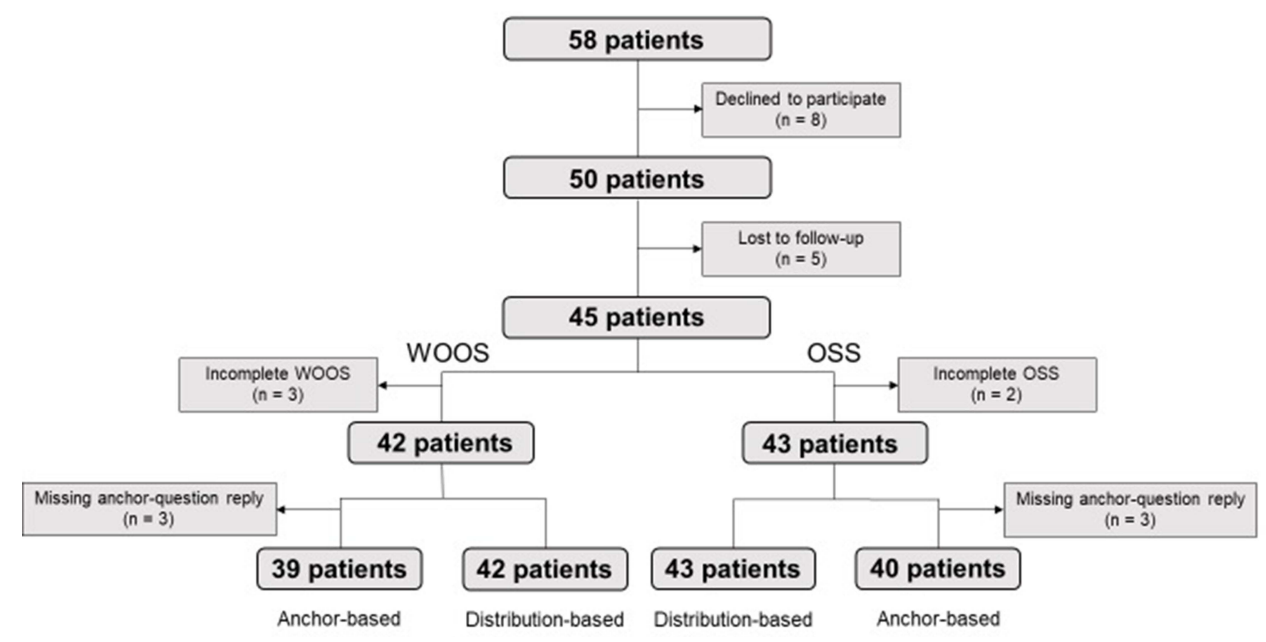

Figure I Flowchart of included patients.

3 patients did not answer the anchor question, leaving 40 and 39 patients, respectively, for the anchor-based analysis (Figure 1).

The grade of glenohumeral osteoarthritis were classified in each patient according to the Walch classification. ${ }^{37}$ The classifications were based on a consensus decision by two experienced shoulder surgeons (Table 2).

The mean preoperative and postoperative WOOS was 33 and 82 respectively, resulting in a mean improvement of 49 points (95\% CI 42.3-56.6, p<0.001) (Table 3). The mean preoperative and postoperative OSS was 22 and 40, respectively, resulting in a mean improvement of 18 points $(95 \% \mathrm{CI}$ 15.5-20.9, $\mathrm{p}<0.001$ ) (Table 3). Mean age at the time of surgery was 65 years (SD 8.76) and $64 \%$ of the patients were women.

\section{WOOS}

\section{Anchor Based Method}

The mean improvement in WOOS was 39.8 in the not importantly changed group and 52.1 in the importantly changed group (Table 4). This resulted in an anchorbased estimate of MCID for WOOS at 12.3.

Table 2 Distribution of Patients According to the Walch Classification

\begin{tabular}{|l|c|c|}
\hline Walch & Frequency & Percent \\
\hline AI & 15 & 33.3 \\
A2 & 6 & 13.3 \\
BI & 6 & 13.3 \\
B2 & 12 & 26.7 \\
C & 6 & 13.3 \\
Total & 45 & 100.0 \\
\hline
\end{tabular}

\section{Standard Error of Measurement}

The standard deviation of the mean preoperative WOOS was 19.1 and Cronbach's $\alpha$ was 0.45. This resulted in the following standard error of measurement, corresponding to an estimate of MCID for WOOS at 14.2:

$$
M C I D=S E M=19.1 \cdot \sqrt{1-0.45}=14.2
$$

\section{Mean Standard Deviation}

Half of the mean SD of WOOS at baseline, follow-up and the difference between baseline and follow-up resulted in an estimate of MCID of 10.3 for WOOS:

$$
M C I D=0.5 \cdot \frac{19.1+19.7+23.1}{3}=10.3
$$

\section{OSS}

\section{Anchor Based Method}

The mean improvement in OSS was 15.4 in the not importantly changed group and 19.7 in the importantly

Table 3 PROM Outcomes

\begin{tabular}{|l|l|c|c|c|}
\hline \multicolumn{2}{|l|}{ PROM $^{\text {a }}$} & Minimum & Maximum & $\begin{array}{c}\text { Mean } \\
\text { (SD) }\end{array}$ \\
\hline WOOS $^{\mathrm{b}}$ & Preoperative & 5.5 & 74.1 & $33.0(19.0)$ \\
$(\mathrm{n}=42)$ & Postoperative & 22.2 & 99.7 & $82.3(19.7)$ \\
& Difference & -0.1 & 89.9 & $49.3(23.1)$ \\
\hline OSS $^{\mathrm{c}}$ & Preoperative & 5 & 37 & $22.2(8.9)$ \\
$(\mathrm{n}=43)$ & Postoperative & 17 & 48 & $40.4(7.6)$ \\
& Difference & -6 & 36 & $18.2(9.0)$ \\
\hline
\end{tabular}

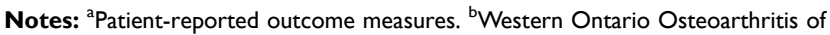
the Shoulder Index. 'Oxford Shoulder Score. 
Table 4 wOOS Outcomes Classified According to AnchorBased Groups

\begin{tabular}{|l|l|c|c|c|}
\hline \multicolumn{2}{|l|}{ Group } & Minimum & Maximum & $\begin{array}{c}\text { Mean } \\
\text { (SD) }\end{array}$ \\
\hline Not & Preoperative & 5.5 & 45.6 & $25.5(13.1)$ \\
importantly & Postoperative & 22.2 & 97.8 & $65.3(23.7)$ \\
changed & Difference & 9.1 & 69.1 & $39.8(19.8)$ \\
$(\mathrm{n}=12)$ & & & & \\
\hline Importantly & Preoperative & 6.1 & 74.1 & $36.0(20.1)$ \\
improved & Postoperative & 46.7 & 99.7 & $88.1(13.4)$ \\
(n=27) & Difference & -0.1 & 84.1 & $52.1(23.4)$ \\
\hline
\end{tabular}

changed group (Table 5). This resulted in an anchor-based estimate of MCID for OSS at 4.3.

\section{Standard Error of Measurement}

The standard deviation of the mean preoperative OSS was 8.9 and Cronbach's $\alpha$ was 0.58 . This resulted in the following standard error of measurement, corresponding to an estimate of MCID for OSS at 5.8:

$$
M C I D=S E M=8.9 \cdot \sqrt{1-0.58}=5.8
$$

\section{Mean Standard Deviation}

Half of the mean SD of OSS at baseline, follow-up and the difference between baseline and follow-up resulted in an estimate of MCID for OSS of 4.3:

$$
M C I D=0.5 \cdot \frac{8.9+7.6+9.0}{3}=4.3
$$

\section{Discussion}

To our knowledge, this is the first study reporting the MCID for WOOS and the first study reporting the MCID for OSS based on patients with glenohumeral osteoarthritis treated with an aTSA.

Table 5 OSS Outcomes Classified According to Anchor-Based Groups

\begin{tabular}{|l|l|c|c|c|}
\hline \multicolumn{2}{|l|}{ Group } & Minimum & Maximum & $\begin{array}{c}\text { Mean } \\
\text { (SD) }\end{array}$ \\
\hline $\begin{array}{l}\text { Not } \\
\text { importantly } \\
\text { changed } \\
(n=13)\end{array}$ & Preoperative & 6 & 35 & $17.9(9.6)$ \\
\hline Postoperative & 17 & 47 & $33.3(8.3)$ \\
Importantly & Pifference & -6 & 33 & $15.4(10.1)$ \\
improved & Preoperative & 5 & 37 & $23.4(8.1)$ \\
& Postoperative & 31 & 48 & $43.2(5.0)$ \\
& Difference & 5 & 36 & $19.7(8.6)$ \\
\hline
\end{tabular}

The assessment and implications of MCID in relation to the PROMs is an important topic in research and in clinical settings. It can be used by researchers in sample size calculations and when results are interpreted, and by clinicians when deciding on individual patient's treatment. In the future, it is no longer necessary to use MCID values for OSS based on other diagnoses, and researchers and clinicians now have a MCID estimate for WOOS that can be used for patients with a shoulder arthroplasty for glenohumeral osteoarthritis.

The MCID value have been reported to differ between pathologies and subgroups of patients. ${ }^{38}$ Therefore, it is important to clarify, that the MCID values calculated in this study can only be used for patients treated with an aTSA for osteoarthritis. For other diagnoses and treatments, the results from this study should be used carefully.

As no previous MCID for WOOS has been reported, our estimates cannot be cross-checked. Thus, we suggest to use 12.3 points as the MCID for WOOS. However, other kinds of metrics to measure clinical improvements have been reported. Newly published data on $\mathrm{SCB}^{15}$ on WOOS indicates that patients need to improve between 45.5 and 53.3 points to be considered as substantial clinical better. And a study by Sciascia et al ${ }^{39}$ reported that the minimum postoperative score that most correctly identified a patient as satisfied was 18 , corresponding to a converted score of 82 points.

A systematic review ${ }^{14}$ reported a mean MCID for OSS at 6 points, ranging from 5 to 6.9 based on four studies. In the present study, the MCID for OSS was 4.3 points for the anchor-based method and 4.3 and 5.8 for the two distribution-based methods. The reason for the lower MCID in our study is speculative but, as previously mentioned, the MCID depends on diagnoses and treatment.

Many methods of estimating MCID have been used and there is no consensus on a "gold standard". ${ }^{2,26}$ If possible, we would have added a second anchor-based method based on a receiver operating characteristic (ROC) curve. The ROC-curve is made by plotting the sensitivity against 1 -specificity at different threshold points corresponding to the improvements in PROM scores. However, this was not possible because of the great improvements in WOOS and OSS. For patients who reported to be only slightly better, the improvement in WOOS was 44.5 and even in those reporting no clinical improvement or worse, the WOOS improved 16 points. Thus, there are virtually no points on a ROC-curve corresponding to the low values of WOOS, and therefore it was 
not possible to determine a MCID using this method. To determine MCID based on a ROC-curve, a much larger population is needed to obtain enough values from the lower end of the ROC-curve.

It is therefore clear, that this study has a major limitation. The small sample size, with only 45 patients in the overall analysis and 39 patients in the anchor-based analysis, may raise concerns on the validity of the results. Nevertheless, the results of this study are the first of their kind and we therefore still consider them to be of high relevance in their function as preliminary data for future studies. In continuation of this study, we therefore highly recommend future studies with much larger populations to validate and cross-check the findings in this study. Additionally, in order to have specific MCID values for other diagnoses and treatments we advocate the MCID to be determined for as many PROMs, with associated subgroups, as possible.

\section{Conclusions}

For patients with glenohumeral osteoarthritis treated with an aTSA the MCID values were 12.3 points for WOOS and 4.3 points for OSS. To our knowledge, this is the first study to report a MCID value for WOOS and the first study to report a MCID value for OSS based on patients with glenohumeral osteoarthritis treated with an aTSA. Therefore, these values lead to a great future benefit for researchers and clinicians in the interpretation of WOOS and OSS. We acknowledge, however, that this is a preliminary study with a small sample size, setting the stage for future studies with much larger populations that may be able to validate data found in this study.

\section{Abbreviations}

PROM, Patient reported outcome measures; MCID, Minimal clinically important difference; aTSA, Anatomical total shoulder arthroplasty; WOOS, Western Ontario Osteoarthritis of the Shoulder Index; OSS, Oxford Shoulder Score; SCB, Substantial clinical benefit.

\section{Data Sharing Statement}

All data is stored in a local archive. In need of access to the data, the corresponding author can be contacted.

\section{Ethics Approval and Informed Consent}

The study is conducted according to the ethics outlined in the Helsinki Declaration. ${ }^{40}$ A permission to handle and store data has been obtained from the Danish Data Protection Agency (j.no.: 2012-58-0004). The study was evaluated by the regional Research Ethics Committee and it was decided that the study did not need approval (j. no.: $\mathrm{H}-17003344$ ). All patients have given informed consent prior to participation. The Ethics Committee/Institutional Review Board is from "Region Hovedstaden". "Region Hovedstaden" is the overall organization that manages all hospitals in the Capital Region of Denmark, including Herlev and Gentofte Hospital, to which the authors are affiliated.

\section{Funding}

DePuy Synthes (Raynham, Massachusetts, United States of America) gave institutional support to the study "Functional outcome and complications after Global Unite prostheses" which provided data for the present study. The funder had no role in the design of the study, execution, analysis, or interpretation, and the funder have no influence on the decision to submit the results.

\section{Disclosure}

Bo Sanderhoff Olsen, Alexander Amundsen and Jeppe Vejlgaard Rasmussen received institutional support from DePuy Synthes for conducting the study "Functional outcome and complications after Global Unite prostheses" which provided data for the present study. In addition, Bo Sanderhoff Olsen and Jeppe Vejlgaard Rasmussen are paid speakers for DePuy Synthes (Raynham, Massachusetts, United States of America). Jeppe Vejlgaard Rasmussen reports speaker fees from Johnson and Johnson and Zimmer Biomet, outside the submitted work. The authors report no other conflicts of interest in this work.

\section{References}

1. Patrick DL, Burke LB, Powers JH, et al. Patient-reported outcomes to support medical product labeling claims: FDA perspective. Value Health. 2007;10(Suppl 2):S125-137. doi:10.1111/j.1524-4733.2007.00 275.x

2. Christiansen DH, Frost P, Falla D, Haahr JP, Frich LH, Svendsen SW. Responsiveness and minimal clinically important change: a comparison between 2 shoulder outcome measures. J Orthop Sports Phys Ther. 2015;45(8):620-625. doi:10.2519/jospt.2015.5760

3. Rasmussen JV, Amundsen A, Sørensen AKB, et al. Increased use of total shoulder arthroplasty for osteoarthritis and improved patientreported outcome in Denmark, 2006-2015: a nationwide cohort study from the Danish Shoulder Arthroplasty Registry. Acta Orthop. 2019;90(5):489-494. doi:10.1080/17453674.2019.1633759

4. Rampal R, Cowling PD. Outcomes of shoulder surgery. Orthop Trauma. 2018;32(3):200-206. doi:10.1016/j.mporth.2018.03.007 
5. Wright MA, Keener JD, Chamberlain AM. Comparison of clinical outcomes after anatomic total shoulder arthroplasty and reverse shoulder arthroplasty in patients 70 years and older with glenohumeral osteoarthritis and an intact rotator cuff. J Am Acad Orthop Surg. 2020;28(5):e222-e229. doi:10.5435/JAAOS-D-19-00166

6. Fevang B-TS, Lygre SHL, Bertelsen G, Skredderstuen A, Havelin LI, Furnes O. Good function after shoulder arthroplasty. Acta Orthop. 2012;83(5):467-473. doi:10.3109/17453674.2012.720118

7. Gallacher S, Williams HLM, King A, Kitson J, Smith CD, Thomas WJ. Clinical and radiologic outcomes following total shoulder arthroplasty using arthrex eclipse stemless humeral component with minimum 2 years' follow-up. J Shoulder Elbow Surg. 2018;27(12):2191-2197. doi:10.1016/j.jse.2018.05.039

8. Rasmussen JV, Olsen BS. The Danish Shoulder Arthroplasty Registry. Obere Extremität. 2019;14(3):173-178. doi:10.1007/ s11678-019-0524-2

9. Ödquist M, Hallberg K, Rahme H, Salomonsson B, Rosso A. Lower age increases the risk of revision for stemmed and resurfacing shoulder hemi arthroplasty. Acta Orthop. 2018;89(1):3-9. doi:10.1080/17453674.2017.1411081

10. AOA PROMs Pilot Project Final Report. Australian Orthopaedic Association National Joint Replacement Registry; 2020. Available from: https://aoanjrr.sahmri.com/documents/10180/681914/AOANJRR +PROMs+Pilot+Final+Report. Accessed September 9, 2021.

11. 16th Annual Report 2019. National Joint Registry for England, Wales, Northern Ireland and the Isle of Man.; 2019:248. Available from: https:// reports.njrcentre.org.uk/Portals/0/PDFdownloads/NJR\%2016th\% 20Annual\%20Report\%202019.pdf. Accessed September 9, 2021.

12. Boyle MJ, Youn S-M, Frampton CMA, Ball CM. Functional outcomes of reverse shoulder arthroplasty compared with hemiarthroplasty for acute proximal humeral fractures. J Shoulder Elbow Surg. 2013;22(1):32-37. doi:10.1016/j.jse.2012.03.006

13. LROI Annual Report 2019. Dutch Arthroplasty Register (LROI); 2019:157. Available from: https://www.lroi-report.nl/app/uploads/ 2020/10/PDF-Online-LROI-annual-report-2019-min.pdf.Accessed September 14, 2021.

14. Jones IA, Togashi R, Heckmann N, Vangsness CT. Minimal clinically important difference (MCID) for patient-reported shoulder outcomes. J Shoulder Elbow Surg. 2020;29(7):1484-1492. doi:10.1016/j.jse. 2019.12.033

15. Razmjou H, Rahnama L, Holtby R, Drosdowech D, Richards R. Substantial clinical benefit, responsiveness, and sensitivity to change of three common outcome measures following shoulder arthroplasty. SAGE Open Med. 2020;8:205031212094621. doi:10.1177/ 2050312120946218

16. Christie A, Dagfinrud H, Garratt AM, Ringen Osnes H, Hagen KB. Identification of shoulder-specific patient acceptable symptom state in patients with rheumatic diseases undergoing shoulder surgery. J Hand Ther. 2011;24(1):53-60; quiz 61. doi:10.1016/j.jht.2010.10.006

17. van Kampen DA, Willems WJ, van Beers LWAH, Castelein RM, Scholtes VAB, Terwee CB. Determination and comparison of the smallest detectable change (SDC) and the minimal important change (MIC) of four-shoulder patient-reported outcome measures (PROMs). J Orthop Surg Res. 2013;8:40. doi:10.1186/1749-799X8-40

18. Ekeberg OM, Bautz-Holter E, Tveitå EK, Keller A, Juel NG, Brox JI. Agreement, reliability and validity in 3 shoulder questionnaires in patients with rotator cuff disease. BMC Musculoskelet Disord. 2008;9 (1):68. doi:10.1186/1471-2474-9-68

19. Lo IK, Griffin S, Kirkley A. The development of a disease-specific quality of life measurement tool for osteoarthritis of the shoulder: the Western Ontario Osteoarthritis of the Shoulder (WOOS) index. Osteoarthritis Cartilage. 2001;9(8):771-778. doi:10.1053/joca.2001.0474
20. Guillemin F, Bombardier C, Beaton D. Cross-cultural adaptation of health-related quality of life measures: literature review and proposed guidelines. J Clin Epidemiol. 1993;46(12):1417-1432. doi:10.1016/ 0895-4356(93)90142-n

21. Rasmussen JV, Jakobsen J, Olsen BS, Brorson S. Translation and validation of the Western Ontario Osteoarthritis of the Shoulder (WOOS) index - the Danish version. Patient Relat Outcome Meas. 2013;4:49-54. doi:10.2147/PROM.S50976

22. Dawson J, Fitzpatrick R, Carr A. Questionnaire on the perceptions of patients about shoulder surgery. J Bone Joint Surg Br. 1996;78 (4):593-600. doi:10.1302/0301-620X.78B4.0780593

23. Frich LH, Noergaard PM, Brorson S. Validation of the Danish version of Oxford Shoulder Score. Dan Med Bull. 2011;58(11):A4335.

24. Goutallier D, Postel JM, Bernageau J, Lavau L, Voisin MC. Fatty muscle degeneration in cuff ruptures. Pre- and postoperative evaluation by CT scan. Clin Orthop Relat Res. 1994;304:78-83.

25. Fuchs B, Weishaupt D, Zanetti M, Hodler J, Gerber C. Fatty degeneration of the muscles of the rotator cuff: assessment by computed tomography versus magnetic resonance imaging. J Shoulder Elbow Surg. 1999;8(6):599-605. doi:10.1016/s1058-2746(99)90097-6

26. Brozek JL, Guyatt GH, Schünemann HJ. How a well-grounded minimal important difference can enhance transparency of labelling claims and improve interpretation of a patient reported outcome measure. Health Qual Life Outcomes. 2006;4:69. doi:10.1186/14777525-4-69

27. Revicki D, Hays RD, Cella D, Sloan J. Recommended methods for determining responsiveness and minimally important differences for patient-reported outcomes. J Clin Epidemiol. 2008;61(2):102-109. doi:10.1016/j.jclinepi.2007.03.012

28. Simovitch R, Flurin P-H, Wright T, Zuckerman JD, Roche CP. Quantifying success after total shoulder arthroplasty: the minimal clinically important difference. J Shoulder Elbow Surg. 2018;27 (2):298-305. doi:10.1016/j.jse.2017.09.013

29. Tashjian RZ, Deloach J, Porucznik CA, Powell AP. Minimal clinically important differences (MCID) and patient acceptable symptomatic state (PASS) for visual analog scales (VAS) measuring pain in patients treated for rotator cuff disease. J Shoulder Elbow Surg. 2009;18(6):927-932. doi:10.1016/j.jse.2009.03.021

30. Tashjian RZ, Deloach J, Green A, Porucznik CA, Powell AP. Minimal clinically important differences in ASES and simple shoulder test scores after nonoperative treatment of rotator cuff disease. J Bone Joint Surg Am. 2010;92(2):296-303. doi:10.2106/JBJS.H.01296

31. Kukkonen J, Kauko T, Vahlberg T, Joukainen A, Aärimaa V. Investigating minimal clinically important difference for constant score in patients undergoing rotator cuff surgery. $J$ Shoulder Elbow Surg. 2013;22(12):1650-1655. doi:10.1016/j.jse.2013.05.002

32. Tashjian RZ, Hung M, Keener JD, et al. Determining the minimal clinically important difference for the American Shoulder and Elbow Surgeons score, Simple Shoulder Test, and visual analog scale (VAS) measuring pain after shoulder arthroplasty. J Shoulder Elbow Surg. 2017;26(1):144-148. doi:10.1016/j.jse.2016.06.007

33. Wyrwich KW. Minimal important difference thresholds and the standard error of measurement: is there a connection? J Biopharm Stat. 2004;14(1):97-110. doi:10.1081/BIP-120028508

34. Wyrwich KW, Nienaber NA, Tierney WM, Wolinsky FD. Linking clinical relevance and statistical significance in evaluating intra-individual changes in health-related quality of life. Med Care. 1999;37(5):469-478. doi:10.1097/00005650-199905000-00006

35. Pornsuriyasak P, Thungtitigul P, Kawamatawong T, Birring SS, Pongmesa T. Minimal clinically important differences (MCIDs) of the Thai Version of the Leicester cough questionnaire for subacute and chronic cough. Value Health Reg Issues. 2017;12:57-62. doi:10.1016/j.vhri.2017.03.009 
36. Norman GR, Sloan JA, Wyrwich KW. Interpretation of changes in health-related quality of life: the remarkable universality of half a standard deviation. Med Care. 2003;41(5):582-592. doi:10.1097/ 01.MLR.0000062554.74615.4C

37. Walch G, Badet R, Boulahia A, Khoury A. Morphologic study of the glenoid in primary glenohumeral osteoarthritis. $J$ Arthroplasty. 1999;14(6):756-760. doi:10.1016/s0883-5403(99) 90232-2

38. Holmgren T, Oberg B, Adolfsson L, Björnsson Hallgren H, Johansson K. Minimal important changes in the Constant-Murley score in patients with subacromial pain. J Shoulder Elbow Surg. 2014;23(8):1083-1090. doi:10.1016/j.jse.2014.01.014
39. Sciascia AD, Morris BJ, Jacobs CA, Edwards TB. Responsiveness and internal validity of common patient-reported outcome measures following total shoulder arthroplasty. Orthopedics. 2017;40(3):e513e519. doi:10.3928/01477447-20170327-02

40. WMA Declaration of Helsinki - ethical principles for medical research involving human subjects; September 7, 2018. Available from: https:/www.wma.net/policies-post/wma-declaration-ofhelsinki-ethical-principles-for-medical-research-involving-humansubjects/. Accessed December 20, 2019.

\section{Publish your work in this journal}

Patient Related Outcome Measures is an international, peer-reviewed, open access journal focusing on treatment outcomes specifically relevant to patients. All aspects of patient care are addressed within the journal and practitioners from all disciplines are invited to submit their work as well as healthcare researchers and patient support groups.

Submit your manuscript here: http://www.dovepress.com/patient-related-outcome-measures-journal
The manuscript management system is completely online and includes a very quick and fair peer-review system. Visit http://www. dovepress.com/testimonials.php to read real quotes from published authors. 